\title{
Optimization of the active MHD spectroscopy system on JET for the excitation of individual intermediate and high- $n$ Alfvén eigenmodes
}

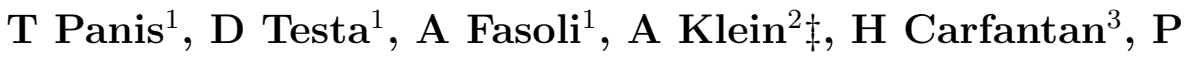 \\ Blanchard $^{1,4}$ and JET-EFDA Contributors $\S$
}

\author{
JET-EFDA, Culham Science Centre, OX14 3DB, Abingdon, UK \\ ${ }^{1}$ Ecole Polytechnique Fédérale de Lausanne (EPFL), Centre de Recherches en \\ Physique des Plasmas, Association EURATOM - Confédération Suisse, CH-1015, \\ Lausanne, Switzerland \\ ${ }^{2}$ MIT Plasma Science and Fusion Center, Cambridge, MA 02139, USA \\ ${ }^{3}$ Laboratoire d'Astrophysique de Toulouse - Tarbes, Université de Toulouse - CNRS, \\ Toulouse, France \\ ${ }^{4}$ EFDA-CSU, Culham Science Centre, OX14 3DB, Abingdon, UK \\ E-mail: theodoros.panis@epfl.ch
}

\begin{abstract}
The stability of Alfvén eigenmodes (AEs) is studied experimentally in the JET tokamak by observing the plasma response to antenna-driven frequency-sweeping perturbations at the plasma edge. During the 2008/9 experimental campaigns, the complete set of the new antennas was operated and AEs with toroidal mode numbers $(n)$ in the intermediate- $n$ range were excited under various plasma conditions. In this paper, we describe the results of the work achieved on the technical aspects of the diagnostic. The antenna currents have been optimized to improve the antenna-plasma coupling. The mode-tracking system has been upgraded for real-time targeting of modes with specific $n$. As an example of the optimized performance of the diagnostic, the paper concludes with a report on the damping rates of $n=3-5$ toroidal AEs (TAEs) that were measured dynamically while the background plasma parameters were evolving in time.
\end{abstract}

PACS numbers: 52.35.Bj, 52.40.Fd, 84.37.+q, 88.80.hp

Keywords: Alfvén eigenmodes, electrical impedance, spectral analysis.

$\ddagger$ Present address: Boston, USA

$\S$ See the Appendix of F. Romanelli et al., Proceedings of the $22^{\text {nd }}$ IAEA Fusion Energy Conference, Geneva, Switzerland, 2008 


\section{Introduction}

The interaction between energetic particles and Alfvén eigenmodes (AEs) [1,2] in tokamak plasmas has stimulated an intense experimental and theoretical research over the last two decades. This combined research resulted in a comprehensive understanding of the various driving and damping mechanisms of AEs. However, the impact of such an interaction in a burning plasma regime, such as the one that will be sustained in ITER and in future fusion reactors, is still unclear [3,4].

Because of the inability to create burning plasma conditions in the current devices, the above question has to be tackled indirectly by enhancing and testing the predictive capability of the theory, via direct comparisons with present day experimental observations. Such studies have already taken place on JET by measuring damping rates of antenna-driven modes of low toroidal mode numbers $(n)$ [5]. The previous antennas, in the following referred to as the saddle coils, could drive modes up to $n=2$, which was a limitation imposed by their in-vessel geometry. However, both experimental and theoretical evidence show that the AEs that are the most prone to destabilization in future large tokamaks have toroidal mode numbers in the medium and high- $n$ range, $n>5[6,7]$.

Extrapolation of the existing results from low- $n$ to high- $n$ modes is not straightforward because the two classes of modes are characterized by different radial structures. Typically, low- $n$ modes tend to have radially extended structure, while high$n$ modes have a more localized structure. The interaction of AEs with the energetic particles depends critically on such a structure. In order to study experimentally the stability of high- $n$ modes, a new set of antennas, to which we will refer to as AE antennas, has been installed in JET and connected to the existing hardware and software infrastructure of the saddle coil system $[8,9]$.

Two important challenges had to be faced to improve the performance of the active diagnostic with the new antennas. The first is the antenna-plasma coupling: relatively high antenna currents needed to be achieved in order to observe a clear signature of high- $n$ modes in the plasma response. The need for high antenna currents in a broad range of frequencies was addressed by designing matching circuits for specific frequency ranges, based on detailed electrical modeling of the excitation system. By virtue of the linearity of the plasma response with respect to the antenna drive, the gain achieved in the signal-to-noise ratio of the plasma response is equal to the increase of the antenna currents.

The second challenge was related to the real-time mode tracking system, which allows one to follow the evolution of the individual modes as the background plasma parameters evolve. A specific method was developed to insure that the tracking algorithm would not lock onto low- $n$ modes and that the algorithm would follow the same $n$ number. The solution was based on the integration of the SparSpec algorithm in the preexisting real-time software. SparSpec $\|$ is the implementation of a new

|| available at http://www.ast.obs-mip.fr/Softwares 

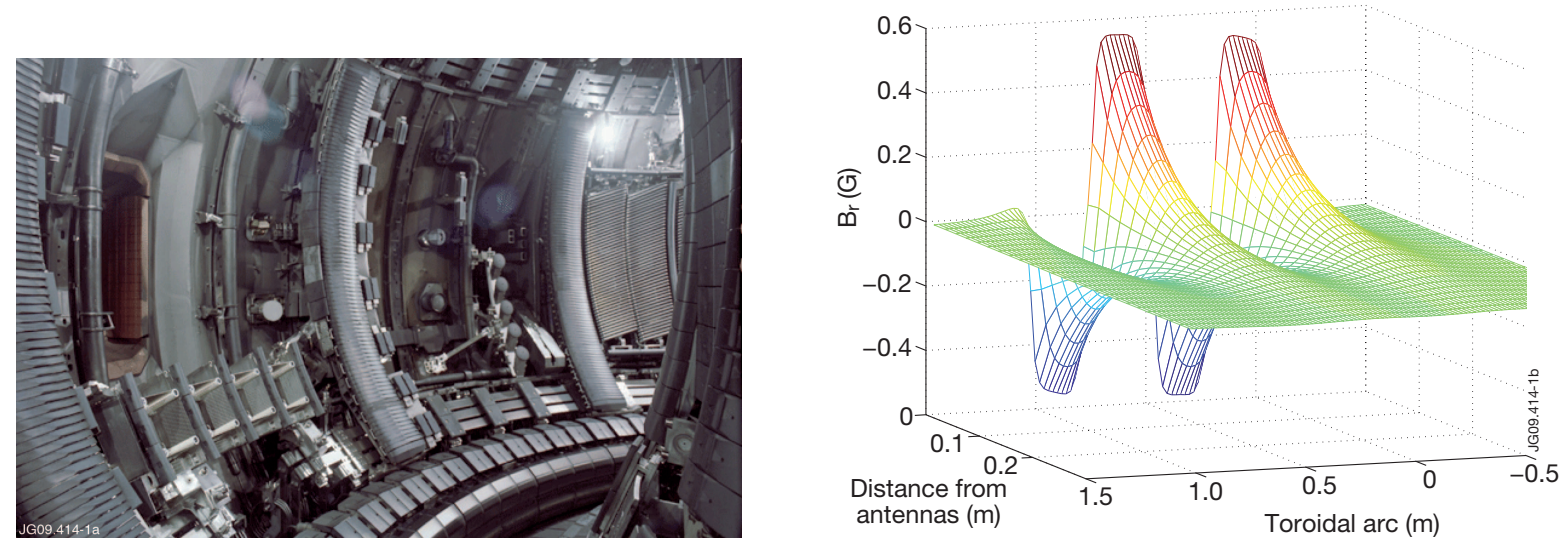

Figure 1: Left- In-vessel view of a 4-antenna array (bottom left). Right- Radial magnetic field produced by a 4-antenna array with +-+- phasing and 1 A current. The field is calculated on a plane extending from the first turn of the antennas towards the torus center.

method of fitting sinusoids to irregularly sampled data [10], based on a sparse spectrum representation of signals. Its performance has been shown to be superior to the other methods of mode number estimation for the post-pulse data analysis [11].

This paper is an extended version of a contribution presented in the $11^{\text {th }}$ IAEA technical meeting on energetic particles [12]. A companion paper [13,14] reports on a quantitative analysis of the damping of medium-n TAEs as function of the edge elongation and the readers are invited to refer to this work for further details. The rest of the paper is organized as follows. Section 2 provides a brief description of the geometry of the antennas and its impact on the mode excitation. Section 3 describes the optimization of the antenna currents from the engineering point of view. The upgrade of the real-time mode tracking software is reported in Section 4 and the first results of the measured damping rates as function of background plasma parameters are presented in Section 5.

\section{The AE antennas}

Eight compact antennas are installed on the low-field-side wall below the $z=0$ midplane at JET. They are divided in two groups of four antennas located at toroidally opposite positions (figure 1, left). Each antenna is a rectangular coil with 18 turns of $4 \mathrm{~mm}$ diameter inconel 718 wire and measures $23 \mathrm{~cm}$ toroidally, $21 \mathrm{~cm}$ poloidally and $14 \mathrm{~cm}$ radially. Adjacent antennas are separated by $7.2 \mathrm{~cm}$. The first turn that faces the plasma is approximately $4.5 \mathrm{~cm}$ behind the poloidal limiters. Each 4-antenna array is mounted on an inconel open frame in order to avoid a closed path for disruption-induced currents.

Using the exact antenna geometry but assuming that the antennas are in vacuum (no plasma, no wall), the antenna field has been calculated. Because of the long 


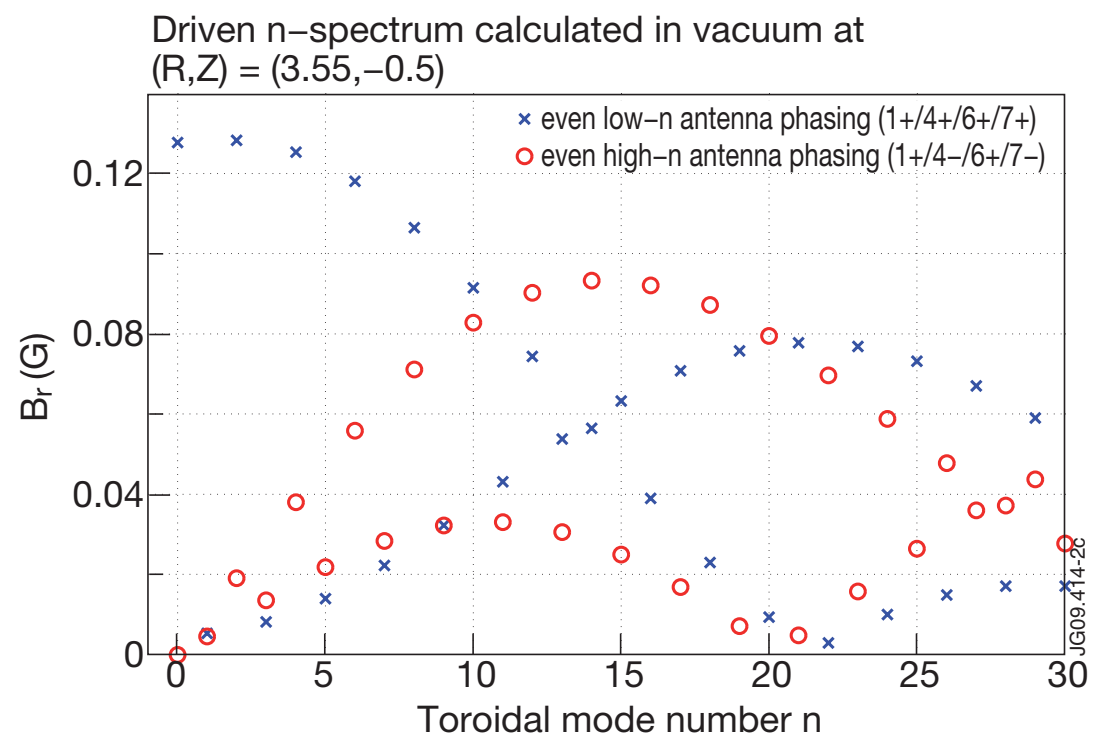

Figure 2: The $n$-spectrum of the antenna radial magnetic field in vacuum using the exact antenna geometry for even-symmetry low- and high- $n$ antenna phasings. Only the right side-band of the spectrum is shown, as the driven $n$-spectrum is symmetric, i.e. the antenna excitation is stationary, comprising of toroidally counter-rotating waves of equal amplitude. The field is calculated at a position close to the last closed flux surface for antenna currents of $1 \mathrm{~A}$.

wavelength $(\lambda>600 \mathrm{~m})$ compared to antenna and the torus dimensions, the antenna field is oscillating harmonically as $e^{i \omega t}$ but it is static in character. At the right of the figure 1, an example of the spatial structure of the radial magnetic field is shown. The field is $0.6 \mathrm{G} / \mathrm{A}$ at the antenna mouth and has an inverse dependence on the distance from the antenna towards the center of the torus. It is less than $0.01 \mathrm{G} / \mathrm{A}$ for $r / a<0.5$ suggesting that the coupling to core-localized modes is very weak.

Based on the field calculation, we can evaluate the $n$-spectrum at any point $[R, Z]$ in the poloidal cross-section for various combinations of the antenna currents. Figure 2 shows the driven $n$-spectra for low and high- $n$ antenna phasing. In figure 2 , the excitation structure is even, giving rise to predominantly even- $n$ components. However, it is also slightly asymmetric because the antennas 1 and 4 do not differ exactly by $\pi$ radians from antennas 6 and 7 respectively. This is why the odd components do not cancel out exactly. Because of the proximity of the antennas along the toroidal angle, the AE antennas can drive a big number of modes with different $n$ that overlap with each other, i.e. the modes could be excited at very close frequencies. This situation is also observed experimentally (an example is shown in figure $5(\mathrm{~b})$ ) and thus constitutes a main motivation for the adaptation of a tool like SparSpec to the mode number analysis for antenna-driven MHD fluctuations, as described in [11]. 


\section{Optimization of the antenna currents}

The layout of the AE excitation system (AE exciter) has remained essentially the same for the new AE antennas, as it used to be during the saddle-coil experiments. It includes a $5 \mathrm{~kW}$ amplifier capable of operating in the range $30-500 \mathrm{kHz}$, an impedance matching circuit, a distribution unit that allows to split the amplifier power into several antennas and to change the antenna phasing, isolation transformers and coaxial cables of more than $100 \mathrm{~m}$ of total length, which transmit the RF power to the in-vessel antennas. However, the impedance of the new antennas is considerably different from that of the saddle-coils: the self-inductance of the new antennas is $70 \mu \mathrm{H}$, while the saddle-coils had an inductance of about $25 \mu \mathrm{H}[15]$.

The impedance characteristics of the AE exciter with the new antennas resulted in a number of problems that were limiting the performance of the active diagnostic. First, it led to relatively low antenna currents (2-3 A peak). These low antenna currents were sometimes insufficient for driving AEs with clear signature on the magnetic pick-up coils, in particular for the excitation of high- $n$ AEs with high- $n$ antenna phasing. Secondly, the strong variation of both amplitude and phase of the antenna currents due to the inductive coupling between the closely-spaced antennas distorts the driven excitation $n$-spectrum and can mislead the real-time mode resonance tracking algorithm into confusing electrical resonances with plasma modes. Hence, it was necessary to optimize the antenna currents, in terms of their amplitude, phase and frequency dependence.

In order to understand the behavior of the antenna currents as a function of frequency and of the system configuration, a detailed electrical circuit model of the AE exciter was built and its validity was confirmed by impedance and voltage/current measurements. The electrical model includes sub-models for the antennas, the distribution-isolation transformers and the transmission lines. Distributed and lumpcomponent representations are used for the transmission lines. For the transformers, high-frequency circuit models with shunt capacitances were constructed from impedance measurements. The inductive coupling between the neighboring antennas has been modeled with mutual inductances calculated from antenna geometry. A very good level of agreement has been achieved between model and measurements and this is shown in figure 3. Using the electrical model, impedance matching circuits were designed and built in order to maximize the antenna currents at different frequency ranges. An analytical description of the electrical model involves technical details which are beyond the scope of the present paper. Here, the final results are reported.

Figure 3 can be used as an example in order to illustrate the constraints imposed by the impedance characteristics of the system. It is observed that the 4-antenna impedance covers a big range from 10 to $700 \Omega$, being thus very different from the nominal value of $50 \Omega$, while the current gain $G(\omega)$ varies by more than 2 orders of magnitude, from 0.01 to 7 . For frequencies higher than $350 \mathrm{kHz}$, the current gain is lower than 0.1 , i.e. a current $I_{\text {dist }}$ of $10 \mathrm{~A}$ results in less than $1 \mathrm{~A}$ in the antennas, while the antenna impedance at the amplifier output is higher than $100 \Omega$. This results in high power reflections into 


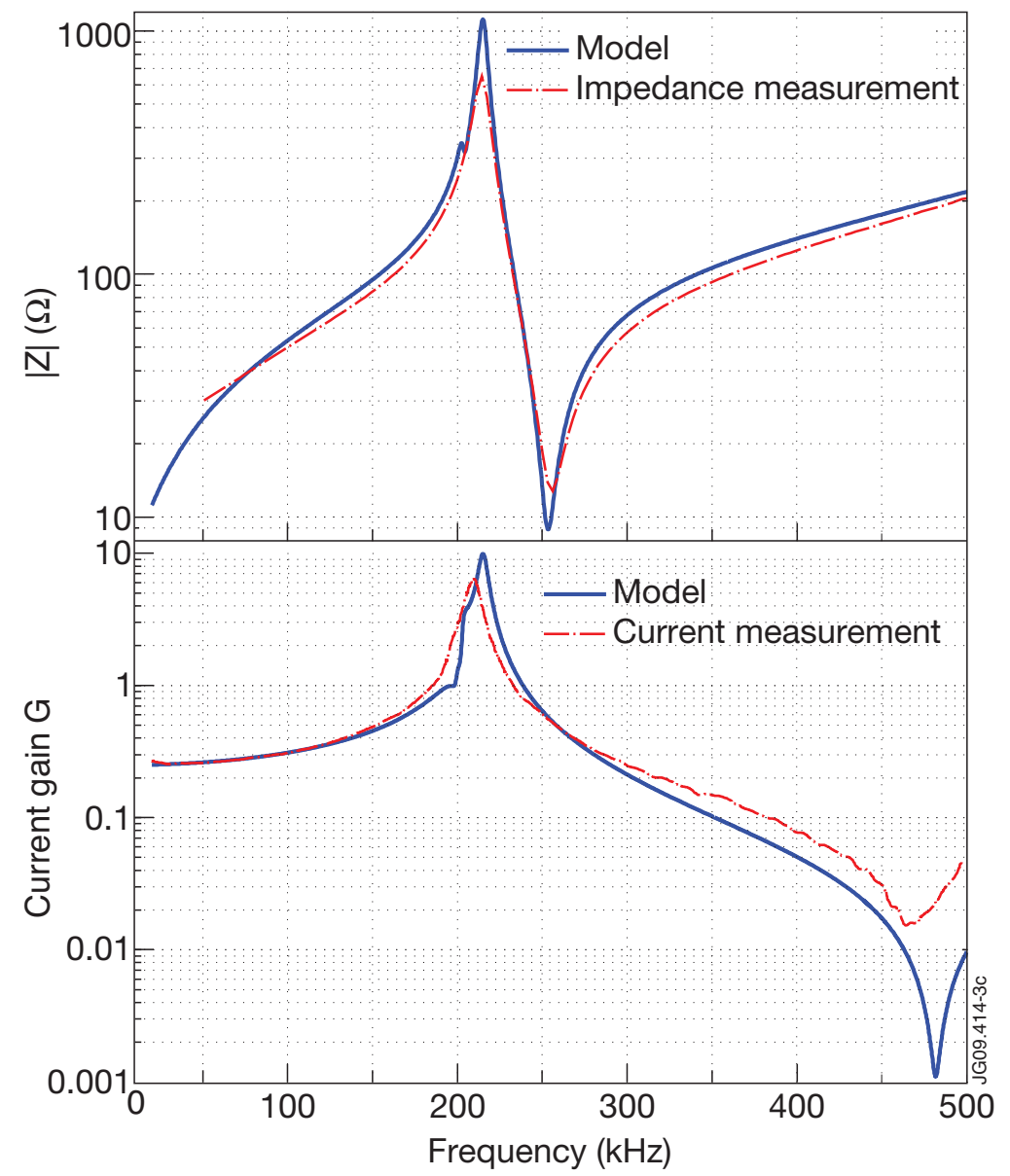

Figure 3: Comparison between the electrical circuit model and impedance/current measurements. The top plot shows the 4-antenna impedance at the distribution unit input, a measurement point close to the amplifier output. The bottom plot shows the current gain $G$, which is defined as the ratio of the antenna current $I_{\text {ant }}$ over the distribution unit input current $I_{\text {dist }}$.

the amplifier and hampers its operation. Thus, part of the typical frequency range of the ellipticity-induced AEs [16] (twice the TAE frequency), as well as the whole frequency range of non-circular AEs [17] (three times the TAE frequency) are not accessible even with modest antenna currents.

Figure 4 shows the measured currents on antenna 7 as a function of frequency, achieved before and after the optimization of the system configuration and with the addition of 2 matching units (MUs). The currents on the other active antennas (not shown here) are of similar magnitude (less than $10 \%$ of difference), because the electrical lengths of the transmission lines are practically equal for all antennas. From a comparison between the antenna currents obtained in the early phase of experiments (beginning of the JET campaign C20, June 2008) and those obtained later (after July 2008), one readily observes that antenna currents almost quadrupled. This increase in the antenna currents was achieved by finely tuning the electronics that control the 


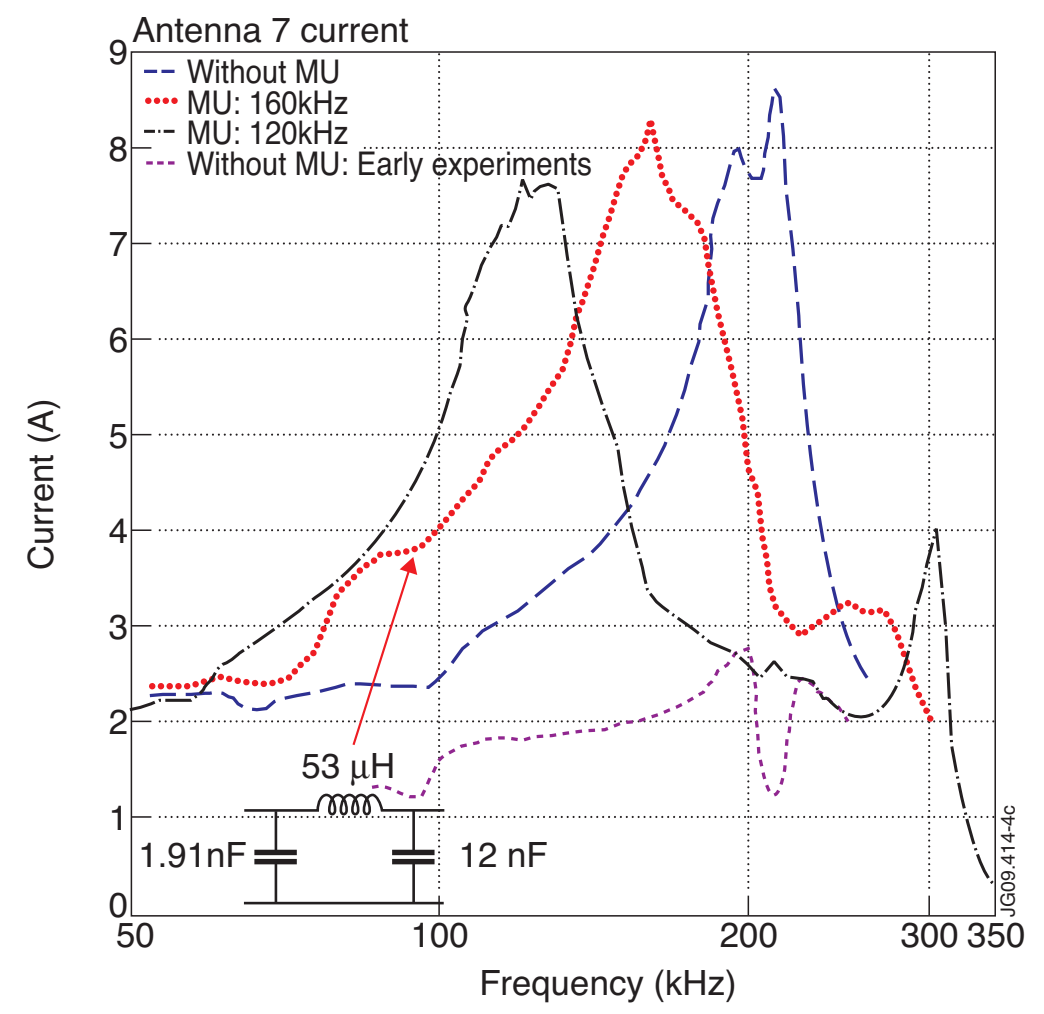

Figure 4: The measured currents on antenna 7 for various exciter configurations, with and without the use of the designed matching units (MUs). The matching circuit used to maximize the antenna currents in the frequency band centered at $160 \mathrm{kHz}$ is also shown.

input signal to the amplifier, based on the understanding provided by the electrical modeling of the excitation system. The increased current obtained around $200 \mathrm{kHz}$ without a matching circuit is due to the fact that the system is naturally matched in that particular range of frequencies. With the addition of 2 matching circuits for frequency bands centered at 120 and $160 \mathrm{kHz}$, the TAE range of frequencies for typical values of the JET magnetic field and density can be efficiently covered with high antenna currents. As shown in figure 4, antenna currents of more than $6 \mathrm{~A}$ (peak) can be achieved from 100 to $230 \mathrm{kHz}$.

The strong variation of the current observed at $210 \mathrm{kHz}$ during the first experiments is due to the strong inductive coupling between the neighboring antennas 6,7 and 8 . The coupling is present even for the other curves shown in the figure, but its effect on the antenna currents is considerably reduced because the contribution from the passive antenna 8 has been minimized. In both situations (before and after the optimization of the system), the antenna 8 is passive, i.e. it is not powered directly by the amplifier because of the disconnected link at the distribution unit. However, current can be driven in the passive antenna by induction from an active antenna (antenna coupling) and it can flow through the distributed capacitance of the coaxial cables which are approximately 
$90 \mathrm{~m}$ long. This coupling is strong when the individual antenna circuits are tuned (see reference [18], page 63), i.e. when they resonate at the same frequency, $200 \mathrm{kHz}$. The coupling from antenna 8 is minimized by detuning the circuit of antenna 8. Detuning is achieved by disconnecting the antenna 8 circuit at a point close to the antenna (just outside the torus). The length of the transmission line of antenna 8 becomes then 20 $\mathrm{m}$ and the resonance frequency increases to $470 \mathrm{kHz}$. Thus the interaction with the neighboring antennas is very much reduced.

The resulting improvement of the antenna-plasma coupling is directly translated into a higher signal-to-noise ratio of the observed plasma response on the Mirnov coils. This achievement is important for the unambiguous determination of the toroidal mode number of the excited modes and the subsequent extraction of their damping rates.

\section{Real-time $n$-decomposition of the plasma response using SparSpec}

Operation of the AE antenna diagnostic is done via the Alfvén Eigenmode Local Manager (AELM). The AELM includes an implementation of a real-time mode tracking algorithm, which was installed and used already in the saddle-coil experiments. In the AELM, the plasma transfer function $H(\omega)=\frac{N(\omega)}{D(\omega)}$ is constructed from the input data, as described in [19]. The input data are synchronously detected signals. Any antenna current can be used for the denominator $D(\omega)$ and the signal from one magnetic probe or the sum of severals of them can be used for the nominator $N(\omega)$. For example, by subtracting the signals coming from two probes located at toroidally opposite position, $N(\omega) \propto 1-e^{i n \pi}$ and the even- $n$ components of the spectrum are filtered out.

This method, although straightforward and robust, offers poor possibilities with respect to filtering specific $n$ numbers. This possibility is now available after the successful integration of SparSpec in the AELM. The implementation of the SparSpec algorithm minimizes the following least-square criterion

$$
J(\mathbf{x})=\frac{1}{2}\|\mathbf{y}-\mathbf{W} \mathbf{x}\|^{2}+\lambda_{N} \max \left|\mathbf{W}^{*} \cdot \mathbf{y}\right| \sum_{k=-K}^{K}\left|x_{k}\right|,
$$

where $\mathbf{y}=\left[y_{1} \ldots y_{P}\right]^{T}$ is the vector of the complex data taken at toroidal angles $\phi_{p}, \mathbf{x}=$ $\left[x_{-K} \ldots x_{K}\right]^{T}$ is the vector of the unknown complex spectral amplitudes associated with mode number $n_{k}, k=-K \ldots K$ and $\mathbf{W}$ is a matrix with elements $W_{p k}=\exp \left(i 2 \pi \phi_{p} n_{k}\right)$. The parameter $\lambda_{N}$ takes values from 0 to 1 and is an input to the minimization algorithm. Higher values of $\lambda_{N}$ make the algorithm converge to solutions with fewer non-zero spectral components. The computational efficiency of SparSpec, which enables its realtime application, is due to the choice of the $\ell^{1}$-norm for the penalization function (second term in equation (1)) and the use of an iterative coordinate descent (ICD) procedure for the minimization of $J(\mathbf{x})$ [10]. With SparSpec, the amplitude-phase information from all magnetic probes is transformed into amplitude-phase spectral information for individual $n$-components. Thus, when AELM is set to use the SparSpec mode of 

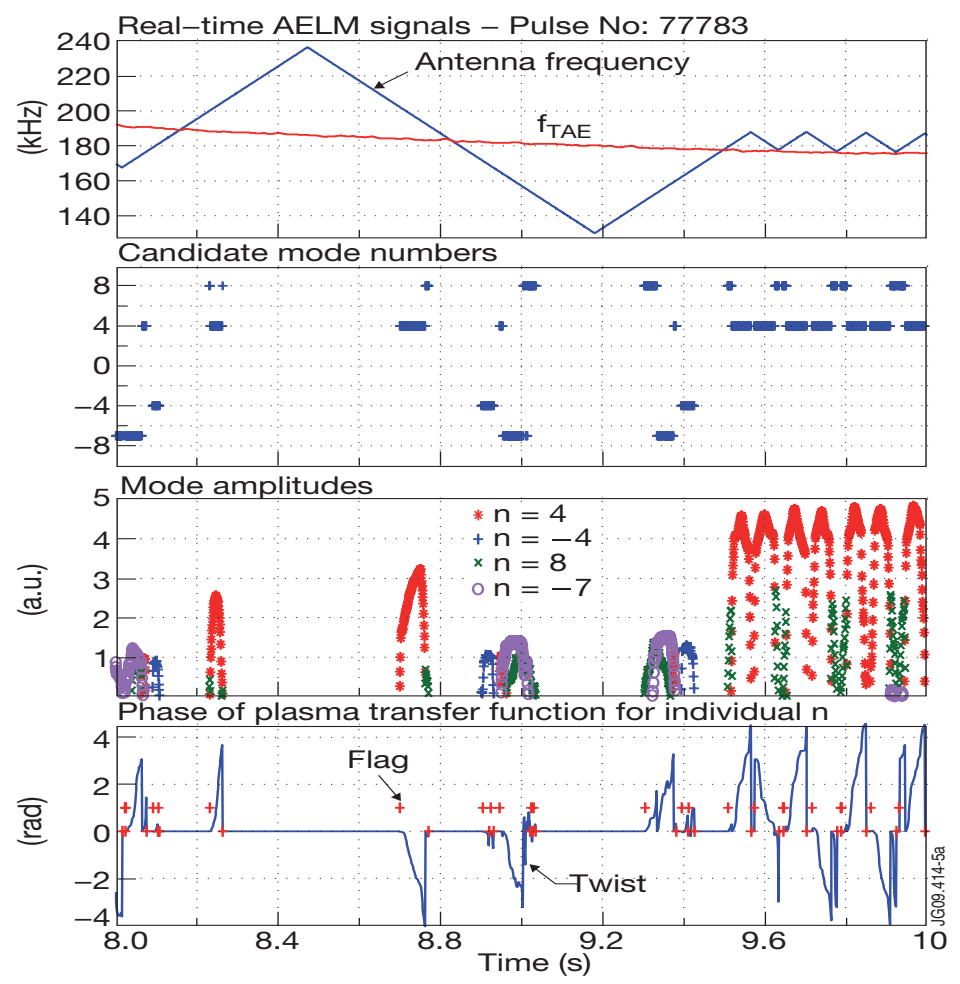

(a)
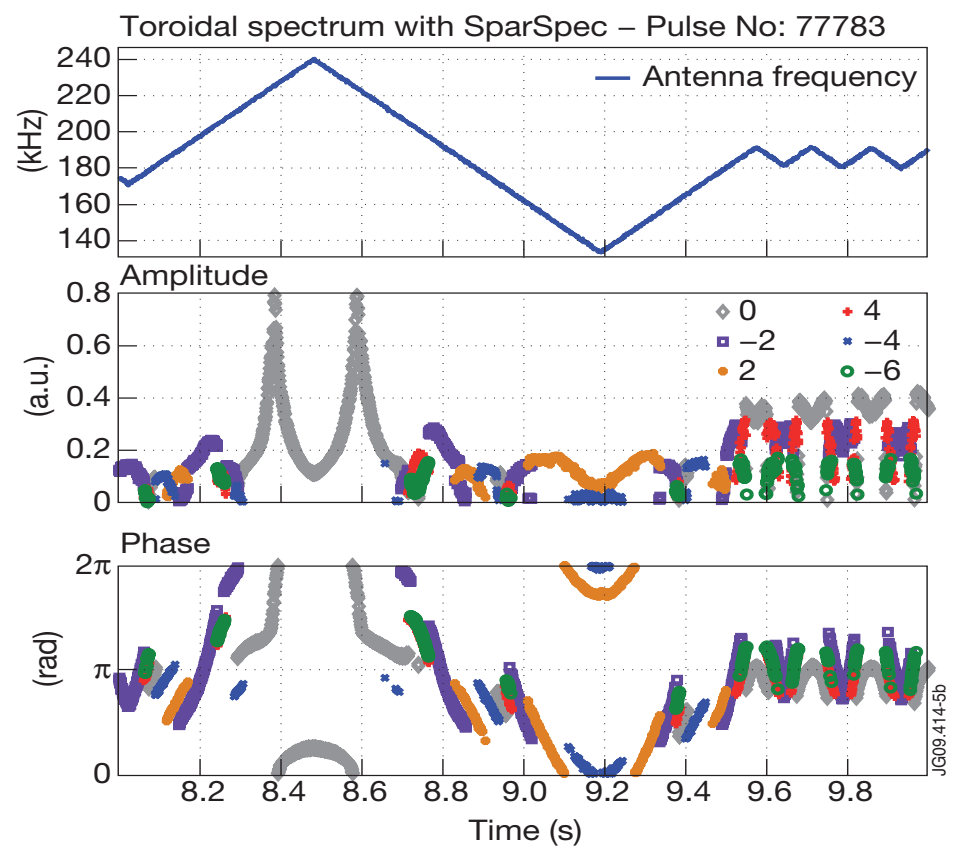

(b)

Figure 5: Illustration of the $n$-number filtering achieved in real-time. The system follows an $n=4$ mode, while several other $n$ s, namely $n=0,-2$ and -6 , are excited inside the narrow frequency scans. (a) Real-time signals of the mode tracking system. The frequency $f_{T A E}=\frac{v_{A}}{4 \pi q R}$ is evaluated in real-time assuming $q=1.5$ and using a lineintegrated interferometry measurement of the plasma core density for calculating the Alfvén speed $v_{A}$. $R$ is torus major radius. (b) Post-pulse estimation of the $n$-spectrum with SparSpec. 
operation, the nominator of the plasma transfer function is specific to an individual mode $n$, i.e. $N(\omega) \propto \delta \dot{B}_{\theta}(n, \omega)$.

The direct benefits from the real-time estimation of the $n$-spectrum are that tracking on low- $n$ modes can be avoided and that tracking on individual (in terms of $n$ ) modes is ensured. Moreover, the detection of simultaneously excited $n$ s by the antennas is possible. An illustration of these capabilities is shown in figure 5. In the top figure 5(a), the real-time signals are shown, while in the bottom figure 5(b), the post-pulse analysis of the data is shown. The user of the diagnostic can choose a set of mode numbers for which tracking is desirable. In this case, a range of $|n| \in[4,8]$ has been enabled. The program estimates the $n$-spectrum and then seeks to identify a mode resonance from the range of $n$ that has been selected. In this example, the candidate mode numbers, i.e. the modes found in the plasma response and belonging to the specified range, have $n=4,-4,8$ and -7 . For these modes, when AELM recognizes a candidate mode resonance, the tracking flag is raised and the twist, i.e. the variation of phase of the plasma transfer function, is computed. If the twist exceeds a given threshold and the mode amplitude falls below a given threshold, a full mode resonance has been completed and the frequency scan changes direction. In this case, the algorithm locks onto a $n=4$ resonance at $9.5 \mathrm{~s}$. As shown in the post-pulse $n$-spectrum, the resonances with $n=0$ and $n=2$ are avoided. Without the use of SparSpec, i.e. using directly the magnetic probe signals, the tracking algorithm would lock onto the strong $n=0$ mode and the resonances of higher $n$ appearing in a different frequency band would have not been detected.

The differences between the real-time $n$-spectrum and the post-pulse $n$-spectrum are that modes with $n=8$ and -7 appear in real-time whereas they are not detected in the post-pulse analysis. Instead, a mode with $n=-6$ is present in the post-pulse results. The other modes, namely with $n=0,2$ and -2 , have not been selected in real-time, hence they do not appear as candidate modes. The differences are due to various reasons. First, the real-time calculation uses a fixed-frequency calibration which is not as accurate as the frequency-dependent calibration used in the post-pulse analysis. Secondly, a digital filter is applied on the raw data in real-time, whereas the post-pulse analysis treats the raw data without filtering. Finally, because of the limited processing power in real-time, the size of the mode number grid was set to $K=15$, whereas the post-pulse analysis uses a grid of $K=30$, satisfying thus better the model requirement for a sparse (i.e., a few non-zero components in the $x$ vector) representation of the $n$ spectrum. Hence, the post-pulse result is a more accurate estimation of the spectral content of the plasma response. This conclusion is in agreement with the imposed antenna phasing (all antennas have the same phase), the symmetry of which leads to a predominant drive of even $n$ s (figure 2, even low- $n$ antenna phasing). Despite the previously described differences, the real-time estimation is quite robust, as it finds successfully the same dominant $n$ s that are calculated in the post-pulse analysis.

Recently, a more powerful processor for the real-time calculation was installed and the software was modified to allow for increasing the size of the mode number 


\begin{tabular}{lll}
\hline Name & $\phi^{\circ}{ }^{\circ}$ & Status 2008-2009 \\
\hline T001 & 2.97 & OK \\
H302 & 92.94 & OK \\
H303 & 103.11 & OK \\
H304 & 108.74 & OK \\
T004 & 110.38 & OK \\
T006 & 182.94 & OK\| \\
T008 & 257.10 & OK \\
T009 & 290.37 & OK \\
\hline
\end{tabular}

|| Intermittently available.

Table 1: Summary of the toroidal array of the magnetic pick-up coils used in this work, all located at $[R, Z] \simeq[3.88,1.01] \mathrm{m}$ with a poloidal orientation $108.9^{\circ}$.

grid. Further improvement of the real-time system will include the possibility of using frequency-dependent calibration and having more options for the filtering of the data.

\section{Damping rate measurements of $n=3-5$ TAEs}

During the JET 2008/9 experimental campaigns, the TAE antenna system was operated in more than 700 JET discharges with each discharge providing tens of damping measurements of various $n \mathrm{~s}$. The experimental data span the intermediate- $n$ range from $|n|=3$ to $|n| \sim 11$ TAEs, including also the lower- $n$ range, $|n|=0-2$. Thus,

a damping rate database is currently being compiled that includes more than 10000 damping measurements for different plasma configurations. This database will be used to systematically study the stability of AEs on JET. Good experimental results have been already achieved both in JET dedicated sessions where the plasma configuration was controlled and during parasitic operation when tracking on a mode could be sustained for a sufficient amount of time. Three such examples are presented in this Section.

Before presenting the damping rate measurements, the key elements of the data analysis are described (part of the methodology is also described in reference [11]). In this work, the AE data come from the edge pick-up coils. Table 1 shows the coordinates and status of the coils that have been used. Fluctuation amplitudes as low as $\left|\delta B_{\theta}\right|>5 \cdot 10^{-8} \mathrm{~T}$ can be detected. In order to ascertain the SparSpec estimations of the $n$ number against errors due to hidden periodicities in the toroidal array of the coils or too much noise of the data, the penalization parameter $\lambda$ was varied over a big range of values. Thus, it was verified that for small values of $\lambda$, i.e. when a big number of modes is tolerated, the dominant modes remain the same. Furthermore, SparSpec results have been shown to be in very good agreement with phase fitting calculations, 

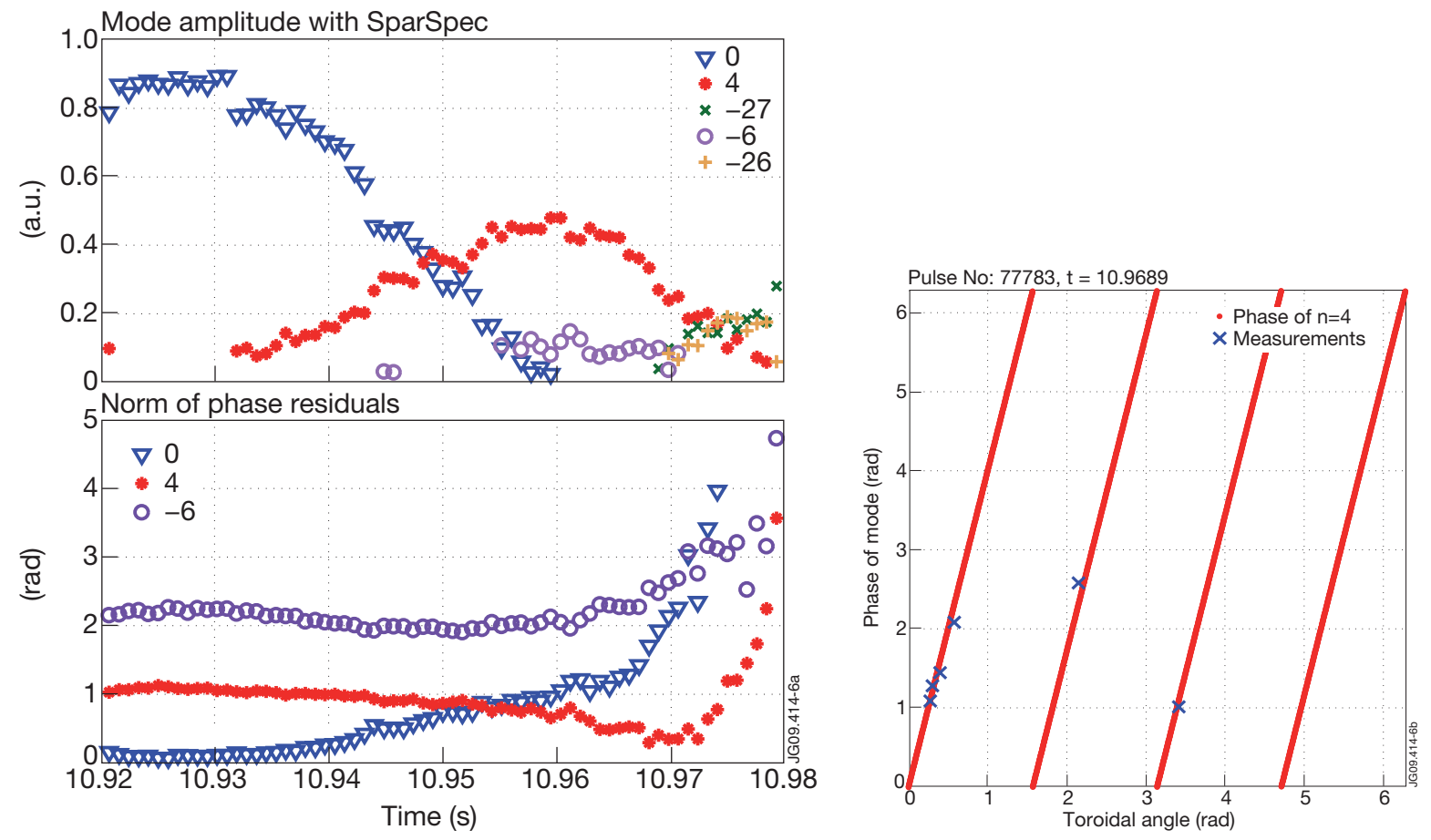

Figure 6: Left- An example taken from JET pulse \#77783 shows modes $n=0$ and 4 overlapping with each other. SparSpec estimation (top) is in very good agreement with the norm of the residuals of the phase fits (bottom). Right- Phases of the magnetic probes at $t=10.9689 \mathrm{~s}$ align very well with the phase of a $n=4$ mode.

as shown in figure 6 .

The data from the magnetic pick-up coils are transformed by SparSpec into amplitudes and phases for individual $n \mathrm{~s}$. The plasma transfer function $H$ is computed separately for each $n$ by taking the ratio of the SparSpec output $N \propto \delta B_{\theta}(n, \omega)$ over the antenna-driven $n$-spectrum produced by the measured antenna currents and calculated according to the expression

$$
D=\tilde{B}_{I}(R, Z ; n) \sum_{j=1}^{8} e^{-i n \alpha_{j}} I_{j}(\omega),
$$

where $\tilde{B}_{I}$ are the current-normalized $n$-components of the radial field produced by one antenna, $\alpha_{j}$ are the antenna coordinates in $\phi$ and $I_{j}$ are the measured antenna currents. This expression allows an exact and quick calculation of the excitation $n$-spectrum without the need to repeat the Fourier calculation for different values of the antenna currents. By fitting the measured transfer function $H=H(n, \omega)$ to a rational function as described in [19], one obtains the frequency and damping rate of individual modes. Such a fit is shown for 2 modes of $n= \pm 5$ in figure 7 .

The JET pulse \#77788 was run during a session dedicated to the stability of intermediate- $n$ AEs with the particular goal of providing experimental data for the comparison with theory in the frame of the International Tokamak Physics Activity 


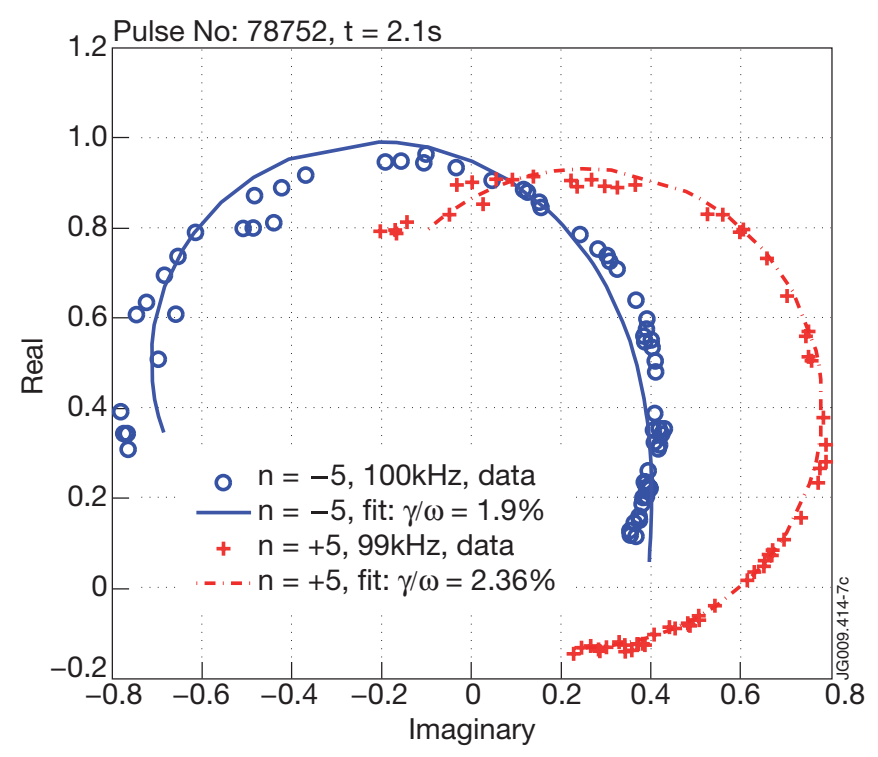

Figure 7: The fit in the complex plane of two simultaneously driven modes $n= \pm 5$, from which the frequency and their damping rate are determined.

(ITPA). As shown in figure 8, the limiter plasma was subject to a very slow edge elongation increase from $t=9 \mathrm{~s}$ to $t=16 \mathrm{~s}$, while the other background plasma parameters were stationary. The damping rates of $n=3$ TAEs were measured several times throughout this phase, allowing to identify a clear increase as a function of the edge elongation. The damping rates $\gamma / \omega$ span the range from $0.3 \%$ at low values of edge elongation to $5 \%$ at high values of edge elongation. Moreover, the increase of the damping rate from $0.3 \%$ to $1 \%$ in the time interval $t=4.5-6 \mathrm{~s}$ when the edge elongation is constant, can be due to the result of the decrease of $q_{0}$, which passes from 1.1 to 0.87 , combined with the increase in density. The observed behavior that damping increases while $q_{0}$ is decreasing is similar to the observations made on the scaling of $n=1$ TAE with $q_{0}$ [21]. The matching unit centered at $160 \mathrm{kHz}$ was used in this pulse.

Figure 9 shows the damping rate measurements extracted from the time window analyzed in Section 4 of the JET pulse \#77783. This discharge comes from the same experimental session as the \#77788 (with the $n=3$ TAEs) and has a similar plasma configuration. Here, we show an extended time window where it can be seen that tracking on the $n=4$ mode continues beyond $t=10 \mathrm{~s}$. Contrary to the $n=3$ mode of the previous example, the damping rate of the $n=4$ mode shows a trend to decrease as function of the edge shear $s_{95}$ as it goes from $1.7 \%$ at $t=8.7 \mathrm{~s}$ to $0.6 \%$ at $t=11 \mathrm{~s}$. This suggests that its damping is affected less or not at all by $s_{95}$, in contrast to the what has been observed for lower- $n$ AEs with the saddle coils $[5,22,23]$ and with the new antennas. However, the variation of the edge shear is small and more measurements are needed to identify its role in the damping. We note here the sensitive dependence of the antenna coupling to the $n=4$ mode on the plasma profiles in these otherwise very stable plasma conditions: by comparing the successive scans of the $n=4$ resonance at 


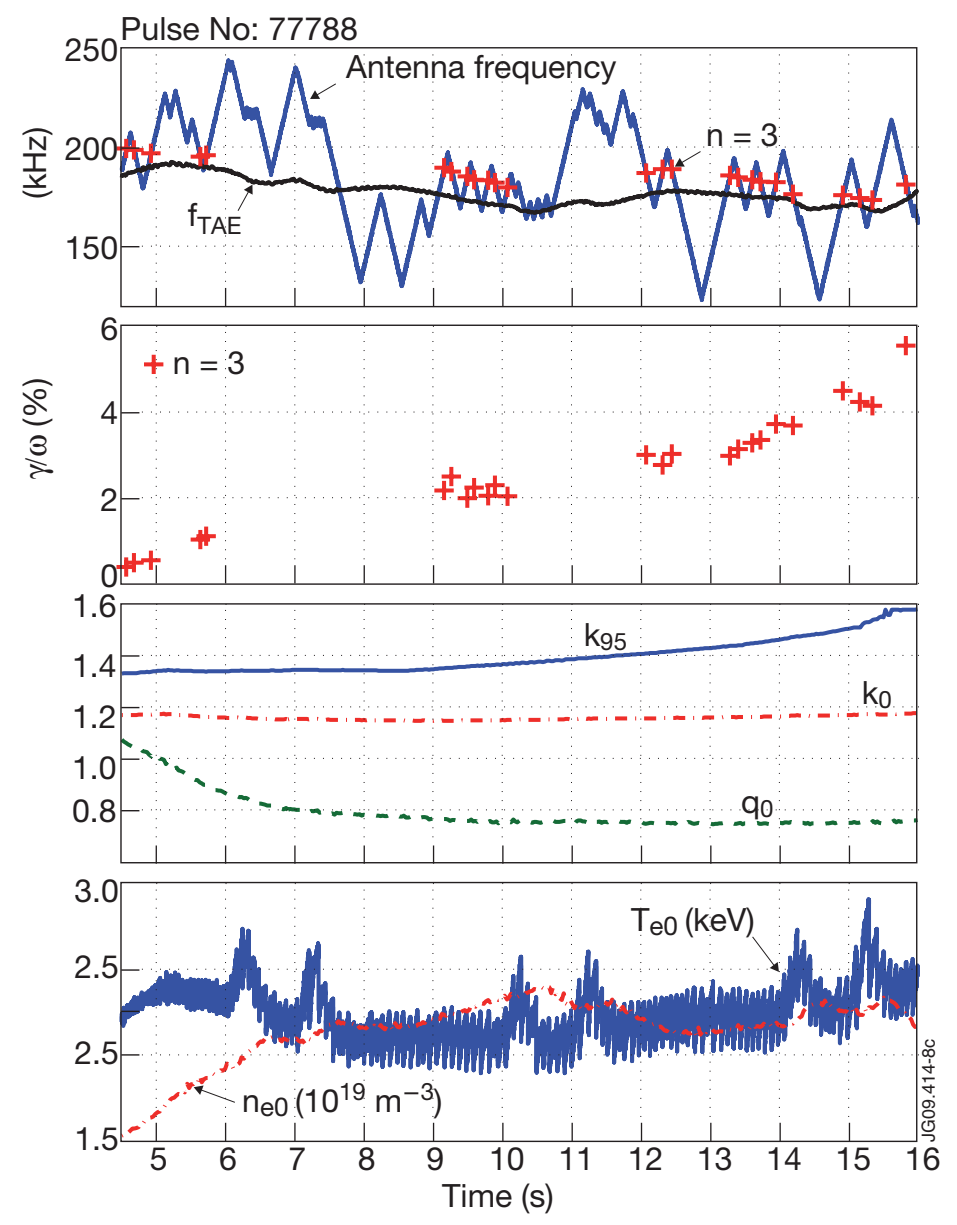

Figure 8: Damping rate measurements of $n=3$ TAEs during an edge elongation scan. Here, $k$ is the elongation and $q$ is the safety factor, $T_{e}$ is the electron temperature and $n_{e}$ is the electron density, with the suffixes ' 0 ' and ' 95 ' indicating core and edge values respectively. The value of $q_{0}$ comes from standard EFIT [20] equilibrium reconstruction and its value has been adjusted by $11 \%$ to match the MSE-constrained equilibrium reconstruction at $t=6 \mathrm{~s}$ and the time of the appearance of sawteeth on the ECE temperature measurements in the plasma core at $t=4.9 \mathrm{~s}$.

$t=8.25,8.75$ and $9.55 \mathrm{~s}$ in figure 5 , one observes a gradual increase both in in the mode amplitude and the number of time points that are resolved into a $n=4$ mode. Damping rate measurements of lower- $n$ AEs are also shown, namely with $n= \pm 2$ and $n=0$. The mode $n=0$ is a global AE [24,25], it has low damping and it is very easily excited in the limiter phase of the plasma discharges, which is a promising result for its use as a diagnostic tool.

Figure 10 shows an example of measurements obtained in the current ramp-up phase of a discharge during parasitic operation. The matching unit centered at 120 $\mathrm{kHz}$ was used in the pulse in order to improve antenna-plasma coupling in the range of low frequencies. The antennas drive simultaneously two TAEs with $n= \pm 5$ at 100 $\mathrm{kHz}$ and the real-time mode tracking is activated causing the antenna frequency to 


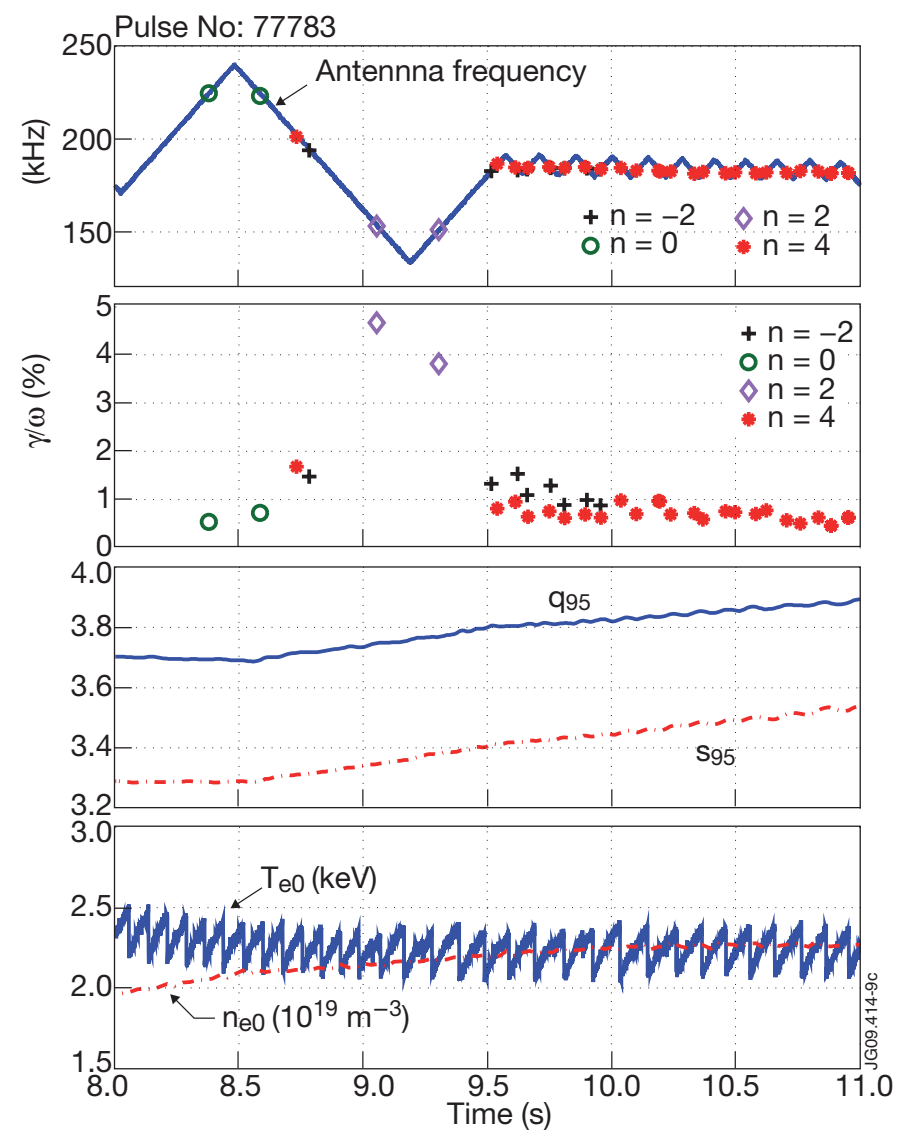

Figure 9: Damping rate measurements of various $n$ s for the case analyzed in Section 3. The damping of an individual TAE with $n=4$ is measured several times by means of the real-time tracking software. Here, $q$ is the safety factor, $s$ is the magnetic shear, $T_{e}$ is the electron temperature and $n_{e}$ is the electron density, with the suffixes ' 0 ' and '95' indicating core and edge values respectively.

scan repeatedly around the mode resonant frequency. During this phase, the monotonic $q$ profile is allowed to settle to lower values while the plasma current penetrates into the plasma core. The safety factor in the plasma core $q_{0}$ remains above 1 during the excitation of the $|n|=5$ modes and this is confirmed by the absence of sawteeth in the ECE temperature measurement in the core. In this time interval, the temperature and density are increasing while the elongation remains constant. The damping rate of the $n= \pm 5$ modes increase from $2 \%$ to $3 \%$. The resonance fits at $t=2.1 \mathrm{~s}$ are shown in figure 7. Similarly to the observations of the previous example regarding the antennaplasma coupling of the $n=4$ TAEs, we note that the detection of $n= \pm 5$ modes is lost at $2.8 \mathrm{~s}$ and that they are not detected at all during the next upward frequency sweep. Instead, $n= \pm 1$ modes are observed. This shows again an increased sensitivity of the antenna-plasma coupling in the intermediate- $n$ range and is indicative of the increased radial localization of the modes, compared to low- $n$ modes. 


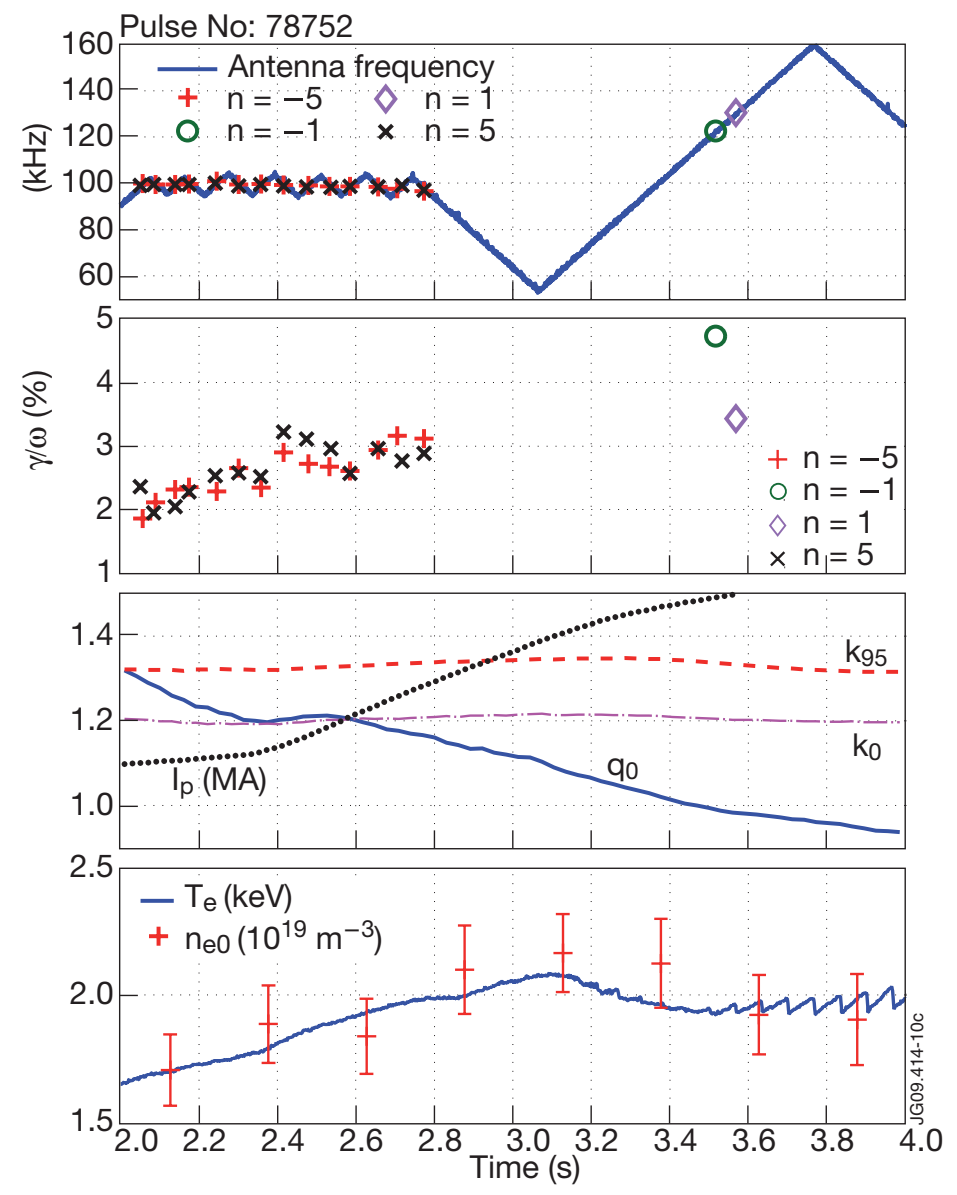

Figure 10: Damping rate measurements of $n= \pm 5$ TAEs in the current ramp-up phase of a discharge. Here, $k$ is the elongation, $q$ is the safety factor, $I_{p}$ is the plasma current, $T_{e}$ is the electron temperature and $n_{e}$ is the electron density, with the suffixes ' 0 ' and ' 95 ' indicating core and edge values respectively.

\section{Conclusions and outlook}

Progress in the technical optimization of the active MHD spectroscopy system at JET has been attained and proved to be important for the achievement of the physics objectives regarding the stability of intermediate- and high- $n$ AEs. High antenna current operation can now cover the TAE range of frequencies with the use of matching circuits, resulting in a significant improvement of the capacity to excite these modes. Filtering of the observed $n$-spectrum in real-time allows one to target individual AEs for mode tracking and measure dynamically their individual damping rates as the background plasma parameters evolve.

The first results from individual discharges that have been presented demonstrate the possibilities of the diagnostic and reveal already information on the stability properties of intermediate- $n$ TAEs. The examples of $n=4$ and $|n|=5$ TAEs indicate that the coupling of the antennas to these modes is more sensitive to the plasma 
profiles than that of low- $n$ modes, suggesting a stronger radial localization. In order to verify such a characteristic of the radial structure of the modes, evidence from internal measurements is being sought. The damping of $n=3$ TAEs was found to increase with edge elongation, suggesting that the control of the AE stability via the edge magnetic shear, conceived for lower- $n$ modes [26], can still apply in the $n=3$ case. The $n=3$ measurements are the subject of a theory-versus-experiment exercise in the framework of ITPA. In a similar discharge, $n=4$ TAEs are found to be less damped than the modes with $n=3$ and less affected by the edge magnetic shear. The damping measurements for the $n= \pm 5$ TAEs during the current ramp-up phase can reveal with more detailed analysis dependence on the profiles of $q$, temperature and density. Analysis of the experimental data is ongoing.

Finally, an upgrade of the system is proposed for the near future within an international collaboration including CRPP and MIT: eight amplifiers could be installed in order to power separately the eight antennas with the capability of setting an arbitrary antenna phasing. This would allow to peak the power of the excitation $n$-spectra in narrow $n$-ranges. It would be also possible to experimentally impose the sign of $n$ and thus, to provide more stringent tests for the comparisons with theory, in particular in the presence of fast ion contribution to the overall mode damping rate.

\section{Acknowledgments}

The Authors would like to thank the various members of the CRPP, MIT and JET staff that have contributed to the design, installation, commissioning and routine operation of the new antenna system. The contribution of C. Bower to the maintenance of the electrical equipment and to the construction of the matching units is gratefully acknowledged. The contribution of A. Goodyear with his work on the integration of SparSpec in the AELM is also gratefully acknowledged.

This work, supported by the European Communities under the contract of Association between EURATOM and CRPP, was carried out within the framework of the European Fusion Development Agreement. The views and opinions expressed herein do not necessarily reflect those of the European Commission.

\section{References}

[1] Heidbrink W W 2008 Phys. Plasmas 15055501

[2] Wong K L 1999 Plasma Phys. Control. Fusion 41 R1

[3] Fasoli A et al. 2007 Nucl. Fusion 47 S264

[4] Chen L and Zonca F 2007 Nucl. Fusion 47 S727

[5] Fasoli A et al. 1997 Plasma Phys. Control. Fusion 39 B287

[6] Heidbrink W W 2002 Phys. Plasmas 92113

[7] Gorelenkov N N et al. 2003 Nucl. Fusion 43594

[8] Fasoli A, Testa D, Sharapov S et al. 2002 Plasma Phys. Control. Fusion 44 B159

[9] Testa D et al. 2004 The New Alfvén Wave Excitation System at JET, Proc. 23 ${ }^{\text {rd }}$ Symposium on Fusion Technology (SOFT) (Venice, Italy) URL http://infoscience.epfl.ch/record/143354/ 
[10] Bourguignon S, Carfantan H and Böhm T 2007 Astronomy \&3 Astrophysics 462379

[11] Klein A et al. 2008 Plasma Phys. Control. Fusion 50125005

[12] Panis T et al. 2009 Optimization of the Active MHD Spectroscopy System at JET for the Excitation of Intermediate and High-n Alfvén Eigenmodes, Proc. 11 $1^{\text {th }}$ IAEA technical meeting on energetic particles in magnetic confinement systems P45 (Kyiv, Ukraine) URL http://infoscience.epfl.ch/record/142715

[13] Testa D et al. 2009 Measurement of the Damping Rate of High- $n$ Toroidal Alfvén Eigenmodes in JET, $11^{\text {th }}$ IAEA technical meeting on energetic particles in magnetic confinement systems $\mathrm{P} 47$ (Kyiv, Ukraine) URL http://infoscience.epfl.ch/record/143355

[14] Testa D, Mellet N, Panis T et al. The Dependence of the Damping Rate of Medium- $n$ Toroidal Alfvén Eigenmodes on the Edge Plasma Elongation in JET. Submitted for publication in this Nucl. Fusion Special Issue

[15] Santaguistina A et al. 1996 Operational experience with the JET saddle coil system, Proc. $19^{\text {th }}$ Symposium on Fusion Technology (Lisbon, Portugal) p 881

[16] Betti R and Freidberg J P 1991 Phys. Fluids B 31865

[17] Betti R and Freidberg J P 1992 Phys. Fluids B 41465

[18] Terman F E 1955 Electronic and Radio Engineering 4th ed (McGraw-Hill)

[19] Fasoli A et al. 1995 Phys. Rev. Lett. 75645

[20] Lao L L, John H S, Stambaugh R D and W P 1985 Nucl. Fusion 251421

[21] Testa D et al. 2005 Nucl. Fusion 45907

[22] Jaun A et al. 1998 Phys. Plasmas 52952

[23] Fasoli A et al. 2000 Phys. Plasmas 71816

[24] Appert K, Gruber R, Troyon F and Vaclavik J 1982 Plasma Phys. 241147

[25] Villard L and Vaclavik J 1997 Nucl. Fusion 37351

[26] Testa D et al. 2001 Nucl. Fusion 41809 